\title{
Le caractère territorial des attractions touristiques liées aux pêches maritimes : une illustration par le modèle du panier de biens
}

\author{
Pocheau Chloé ${ }^{1}$, Alban Frederique ${ }^{1}$, Le Floc'h Pascal ${ }^{1,}{ }^{*}$, Legouvello Raphaela ${ }^{1}$
}

1 Université de Brest, luem, Umr Amure

* Corresponding author : Pascal Le Floc'h, email address : plefloch@univ-brest.fr

\begin{abstract}
:
The question addressed in this article focuses on the conditions for strengthening the territorial character of tourist attractions around a fishing port with a strong identity. In the face of the depletion of resources, the territories dependent on the exploitation of living marine resources try reconversions during the 1990s, especially towards tourism. The article is based on the fishing port of Guilvinec on which has been created an exhibition center for a large audience. The survey methodology is mobilized to delimit the territory on which the fishing activities and the exhibition center are attractive. An estimate of the expenses incurred by visitors is proposed. The tourist's consumption of the exhibition center and fisheries is in line with the basket of goods model.
\end{abstract}

\section{Résume}

La question abordée dans cet article porte sur le caractère territorial des attractions touristiques autour d'un port de pêche à forte identité. Face à l'épuisement des ressources, les territoires dépendants de l'exploitation des ressources marines vivantes tentent au cours des années 1990 des diversifications d'activité, notamment vers le tourisme. L'article retient le cas d'étude du port de pêche du Guilvinec sur lequel est implanté depuis 2000 un centre de découverte destiné à attirer une clientèle touristique. La méthodologie d'enquête est mobilisée pour délimiter le territoire sur lequel s'exerce une attractivité des activités de pêche et du centre de découverte. Une estimation des dépenses engagées par les visiteurs sur ce même territoire est proposée. Le modèle du panier de biens est adapté dans l'étude du comportement de la clientèle touristique. En effet, le centre de découverte offre une combinaison de biens au choix des visiteurs, avec un fort ancrage territorial. 
- 1-

\section{Introduction}

La reconversion des territoires dépendants de l'industrie des pêches maritimes est un thème prioritaire dans les politiques publiques d'aménagement face à l'évidence de la surexploitation des stocks de poissons. C'est au début des années 1990 que les pouvoirs publics créent des programmes de reconversion des flottes de pêche. Au Canada, le moratoire sur la pêche à la morue en 1992 contraint le gouvernement fédéral à créer des fonds spéciaux pour aider les pêcheurs à trouver d'autres sources de revenus (SCHRANK, 2005). En Europe, les plans de réduction du nombre de navires s'accompagnent de pistes de diversification vers des démarches de valorisation de la ressource (CHARLES, 2009), de diversification d'activité (ALBAN et BONCOEUR, 2004) ou de nouvelles formes de régulation (ALBAN et al., 2011). La fonction touristique des activités de pêche est plus rarement exploitée, parfois admise comme un élément de la multifonctionnalité (LESUEUR et al., 2007).

La problématique abordée dans cet article identifie les points d'articulation entre tourisme et pêche maritime, deux industries qui présentent en première analyse peu d'éléments de complémentarité. Certes, les sites littoraux accueillant des ports de pêche plutôt spécialisés sur une activité côtière et artisanale renforcent l'attractivité du territoire et son potentiel touristique (ROPARS-COLLET et al., 2015). Mais les contraintes techniques (manipulation des engins de pêche), logistique (sécurité des sites de débarquement dans des halles à marée) et réglementaires (politiques de gestion des stocks halieutiques complexes) rendent difficiles l'accès d'une population touristique à une meilleure connaissance du monde de la mer et de ses pêcheurs.

La littérature consacrée à l'attractivité des sites touristiques reconnait cependant que l'existence d'un flux important de visiteurs ne repose pas uniquement sur la présence d'une richesse naturelle (paysage exceptionnel unique) ou l'histoire des communautés (culture, activités anciennes). Fautil encore que ces éléments du patrimoine naturel ou culturel soient mis en valeur. GAGNON (2007) évoque la représentation symbolique par des artistes (littérature, peinture) qui interprètent les éléments de la nature dans leurs auvres. La manifestation artistique des métiers de la pêche se trouve dans les œuvres littéraires de Pierre LOTI, photographiques d'Anita CONTI et dans les peintures de Mathurin MEHEUT par exemple. De nombreuses villes côtières célèbrent par des festivités une espèce emblématique : la coquille Saint-Jacques en baie de Saint-Brieuc (LESUEUR et al., 2014), la sardine à Douarnenez (BOUlARD, 1991), la morue dans les ports de Mer du Nord et de la Manche (MORANDIERE, 1967), ou le homard dans l'Etat du Maine aux Etats-Unis (ACHESON, 1975).

On ne peut nier la forte attractivité des ports de pêche auprès d'une clientèle touristique. Toutefois, les difficultés d'accès aux sites portuaires compliquent la création d'une économie du tourisme sur ou à proximité des bassins portuaires. La question abordée dans cet article porte sur le caractère territorial des attractions touristiques autour d'un port de pêche à forte identité. Le cas d'étude retenu est le port du Guilvinec et «Haliotika », le centre de découverte des pêches maritimes situé au cœur du complexe portuaire. Le modèle du panier de biens (PECQUEUR, 2001) offre un support théorique pour décrire la demande touristique attachée à une combinaison de biens complémentaires et dépendants, produits en un même lieu (visite du centre, visite du premier 
marché aux poissons, atelier culinaire, sortie en mer). L'originalité de l'article situe le modèle du panier de biens sur un site localisé au coeur d'un complexe portuaire à vocation artisanale (petite pêche et pêche côtière) et industrielle (pêche au large par une flottille chalutière) ${ }^{1}$, avec une estimation des dépenses touristiques (hébergement et restauration).

Les travaux empiriques sur les modèles de biens composites indiquent un consentement à payer plus élevé des consommateurs quand des externalités territoriales se manifestent. Des domaines aussi variés que la R\&D (FELDMAN and AUDRETSCH, 1999), les industries manufacturières sous formes de districts marshalliens (DAUMAS, 2007), les services tels que le tourisme (BOJAMIC and Calantone, 1990) bénéficient de ces externalités par des effets d'agglomération. Dans cette recherche, la création d'un centre de découverte des pêches maritimes situé sur le site portuaire en activité répond certes à une logique d'activité induite (le tourisme) par une industrie de base (les pêches maritimes). Sans rejeter les apports de la théorie de la base, nous proposons une approche par le consommateur en discutant des différentes formes que revêt le modèle du panier de biens.

La première section apporte des informations sur la définition des territoires liés à l'industrie des pêches maritimes. L'organisation territoriale de cette activité remonte au $17^{\text {ème }}$ siècle, afin de garantir à l'Etat une main d'œuvre formée aux métiers de la mer pour des besoins militaires. La seconde section rappelle qu'une pression de pêche mal régulé a accéléré le phénomène de surexploitation. La manifestation de l'épuisement des stocks a contraint les communautés littorales à une reconversion, dont à titre marginal le tourisme en partie induit par l'activité de base que représentent les pêches maritimes. Le cas d'étude, décrit dans la troisième section, est le quartier maritime du Guilvinec. Ce territoire, comparativement aux autres quartiers maritimes en France, maintient une forte dépendance économique à l'égard des métiers de la pêche. Les emplois de la sphère productive y sont plus élevés que ceux de la sphère présentielle (INSEE, 2015). Les acteurs locaux, conscients d'une érosion continue des emplois à la pêche, ont cherché à profiter d'un flux touristique élevé en créant un centre de découverte au sein même du complexe portuaire. Cette offre cible une clientèle locale mais surtout touristique, attirée par une combinaison de biens leur donnant accès à une connaissance approfondie des métiers de la filière pêche. On retrouve les caractéristiques du modèle de panier de biens revisité à la section quatre. Les données et méthodes sont présentées dans la section cinq en prenant appui sur le modèle du panier de biens. La section six dévoile les résultats produits par l'enquête avec une première estimation des dépenses effectuées sur la commune par les visiteurs du centre de découverte des métiers de la pêche. Les limites et intérêts du travail sont discutés dans la section finale en revisitant le modèle du panier de biens.

\footnotetext{
${ }^{1}$ La classification officielle selon les titres de navigation distingue la petite pêche (durée en mer inférieure à 24 heures), la pêche côtière (entre 24 et 96 heures), la pêche au large (plus de 96 heures en mer), et la grande pêche (plus de 20 jours de navigation).
} 
$-2-$

\section{Le caractère territorial des pêches maritimes}

L'organisation des pêches maritimes est le produit d'une longue histoire débutée sous le règne de LOUIS XIV. Les "gens de mer ", de la pêche et du commerce, ont attiré l'attention des monarchies successives depuis les ordonnances de COLBERT en particulier celle de 1673 (CONFOLENT, 2007), afin de satisfaire aux besoins de la marine de guerre et d'assurer une pension aux familles de marins péris en mer. La population de marins dépend de l'Etablissement national des invalides de la marine (ENIM), une institution formée sous COLBERT pour l'administration du régime social des gens de mer (CORDON, 1950). Le statut de marin pêcheur est accordé aux inscrits maritimes (VAUCLARE, 1987, p249), déclarés auprès du quartier maritime de rattachement, unité territoriale sur laquelle est effectué le recensement des marins.

La vie sociale et économique autour des pêches maritimes s'organise dans les communes portuaires de chaque quartier maritime. La révision générale des politiques publiques (RGPP) a conduit à la suppression officielle des quartiers maritimes au $1^{\text {er }}$ janvier 2010, remplacé par les quartiers d'immatriculation des navires. L'administration des gens de mer regroupé au sein de la Direction Départementale des Territoires et de la Mer - DDTM - conserve cependant les mêmes limites entre l'ancienne et la nouvelle dénomination. On retient dans cet article la dénomination historique. On recense 40 quartiers maritimes en France métropolitaine et 7 dans les départements et territoires d'Outre-mer (figure 1). 
Figure 1 Les quartiers maritimes en France métropole
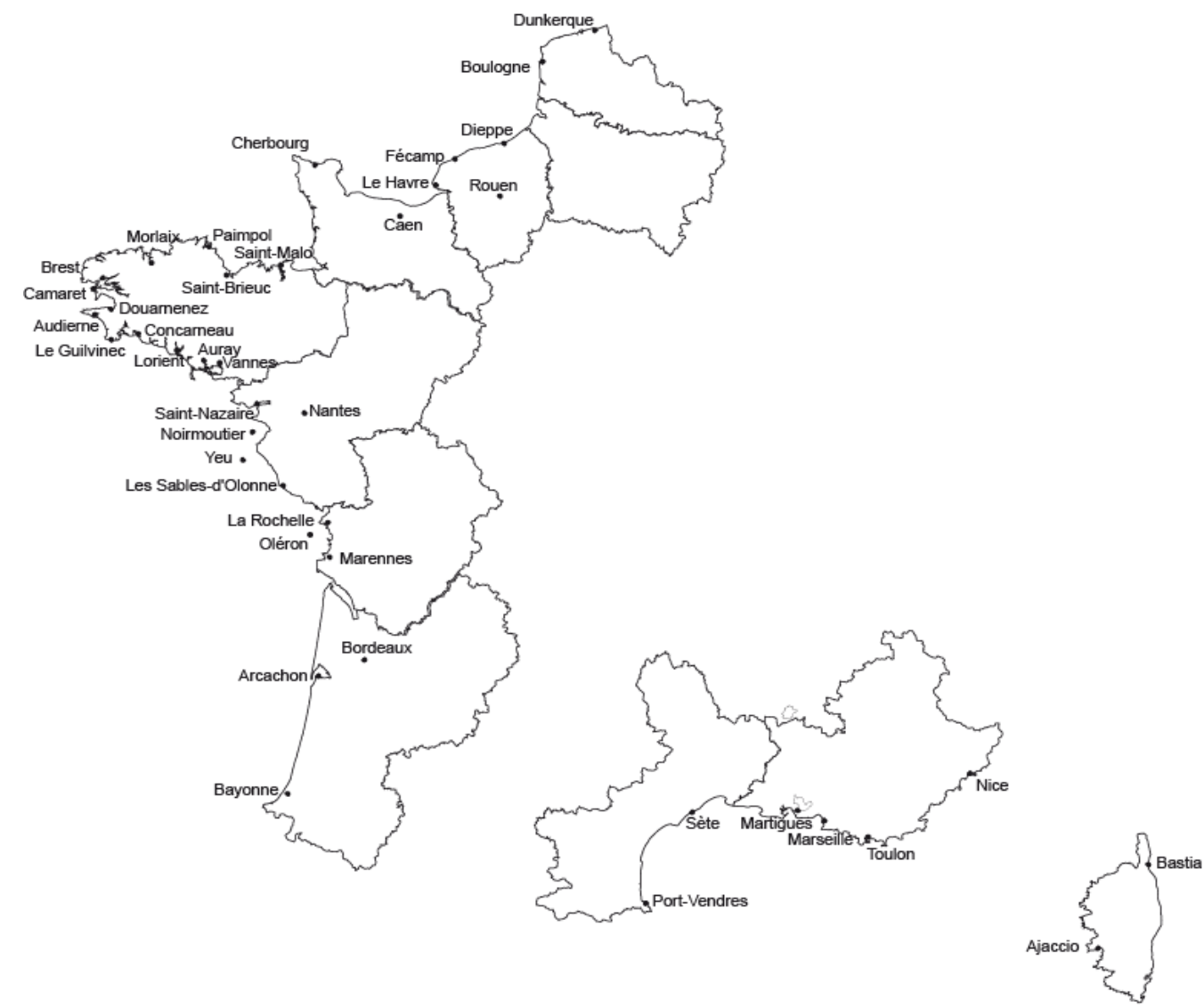

Source : LE FLOC'H et WILSON (2017)

Le caractère territorial des pêches maritimes en France est fortement lié à la présence de pêcheries côtières renforçant l'identité des communautés souvent marquées par des crises (GOUZIEN, 2014 ; DuRAND, 1991). Les ports de la Mer du Nord et de la Manche orientale, de Dunkerque à Granville, accueillent des flottilles exploitant les stocks de maquereau, de plie, de seiche et coquillages. En Bretagne Nord, la pêcherie de coquilles Saint-Jacques en baie de SaintBrieuc est devenu un cas d'école, non seulement dans les sciences marines mais également en sciences humaines et sociales. Le célèbre article de Michel CALLON (CALLON, 1986), sociologue de l'innovation, mobilise la sociologie de la traduction en prenant comme exemple la situation de la pêcherie de coquilles Saint-Jacques de la Baie de Saint-Brieuc. La Méditerranée se démarque des façades maritimes de l'Atlantique et de Manche et Mer du Nord par une flottille essentiellement armée en petite pêche avec un mode de commercialisation sans passage dans une halle à marée (ou criée), à l'exception des quartiers de Port-Vendres, Sète et Martigues.

Face à l'épuisement des ressources, l'ensemble des quartiers doit composer à la fin des années 1980 avec une réduction forte du nombre d'entreprises de pêche. En Europe comme au Canada, l'année 1992 marque un tournant des pêches maritimes. En Amérique du Nord, l'effondrement du stock de morue conduit le Ministère des pêches canadien à appliquer un moratoire en juin 1992, 
mettant près de 20000 personnes de la province de Terre-Neuve au chômage (PARSONS, 1995 ; SCHRANK, 2005). Sur l'archipel de Saint-Pierre et Miquelon, ce moratoire annonce aussi la fin de l'industrie halieutique dans son fonctionnement industriel (LE FLOCH et al., 2017). En Europe, et plus particulièrement dans les ports français, la crise structurelle des pêches maritimes se produit en décembre 1992 sur une durée de près de deux ans (DELBOS ET PREMEL, 1996 ; LE FLOC'H et WILSON, 2017). Les territoires dépendants de l'exploitation des ressources marines vivantes tentent des diversifications d'activité ou des reconversions, notamment vers le tourisme avec l'appui du programme européen Pesca Tourisme.

\section{$-3-$}

\section{Reconversion des territoires maritimes}

La première tentative de reconversion des activités de pêche vers le tourisme, dans le cadre de la Politique commune de la pêche, remonte à 1991. La Commission européenne impose aux Etatsmembres une limitation de la capacité de pêche. La limitation de l'accès prend une forme inédite pour les pêches maritimes françaises. Le Ministre des pêches, Jacques MELLICK, annonce en 1991 le premier plan de sortie de flotte pour atteindre l'objectif de réduction de l'effort de pêche imposé par la Commission européenne aux pays côtiers. Les pêcheurs volontaires au retrait de leur outil de production de la flotte de pêche disposent de trois options, soit la démolition du navire, un transfert définitif vers un pays tiers ou une affectation à d'autres fins que la pêche pour l'embarquement d'une clientèle touristique.

A la clôture du plan en septembre 1991, une puissance motrice globale de près de $100000 \mathrm{KW}$ est retirée, principalement par la démolition ou le transfert vers des pays tiers. $4 \%$ seulement de cette puissance motrice est reconvertie vers d'autres usages que la pêche, notamment le transport de passagers. C'est en Méditerranée que cette option connait le plus de succès, soit un quart des dossiers.

L'ajustement structurel de la taille des flottes de pêche à la disponibilité des ressources s'accélère au cours des années 1990. La reconversion et la diversification des territoires dépendants de la pêche deviennent un thème majeur, accompagné financièrement par des programmes européens, notamment le Fonds européen à la pêche et le Fonds social européen. Les projets d'embarquement d'une population touristique sur des bateaux de pêche se développent, surtout en Méditerranée, en France et en Italie (FOUCAUd, 2008 ; DELAMARE, 2010 ; ALBAN, 1998 ; ALBAN et BONCOEUR, 2004). L'accueil de passagers sur des navires conçus pour une pêche professionnelle impose des aménagements contraignants. Les expériences de diversification menées en agriculture ont aussi un écho dans l'industrie des pêches maritimes. Il s'agit de reconnaitre la multifonctionnalité des activités de la pêche au sein de leur territoire (MERRIEN et al., 2008).

La Commission européenne consacre des moyens financiers au cours des années 1990 pour appuyer des initiatives locales dans le sens d'une reconversion ou d'une diversification des populations dépendantes de la pêche. Il s'agit d'exploiter les interdépendances entre l'activité de base, les pêches maritimes, et l'activité du tourisme en partie induite par la première. Pour dépasser le cadre muséographique des célébrations d'un produit reconnu comme marqueur identitaire du 
territoire (la sardine à Douarnenez, les pêcheurs de morue d'Islande et de Terre-neuve de Dunkerque à Paimpol), la ville du Guilvinec s'est dotée d'un outil de promotion touristique de la pêche dans une démarche commerciale. La prochaine section décrit le cas d'étude du centre de découverte des métiers de la pêche implanté sur le port de pêche du Guilvinec.

\section{$-4-$}

\section{Présentation du cas d'étude : le quartier maritime du Guilvinec}

Le quartier maritime du Guilvinec se compose d'un réseau de quatre ports (Saint-Guénolé, Le Guilvinec, Lesconil et Loctudy). Les productions débarquées sont mises aux enchères dans trois halles à marée (Saint-Guénole, Le Guilvinec et Loctudy). Aucun autre quartier maritime ne réunit autant de structures de débarquements et de lieux de ventes officiels intégrés au réseau statistique national (FRANCEAGRIMER, 2014). Le choix du cas d'étude se porte sur ce quartier maritime compte tenu de son importance à l'échelle nationale et d'une initiative territoriale basée sur les interdépendances entre pêche et tourisme.

Sur les trente dernières années, les écarts de production se sont fortement réduits entre les quartiers maritimes de métropole (LE FLOC'H et WILSON, 2017). Dix d'entre eux dépassaient les 10000 tonnes en 1983, dont quatre à plus de 30000 tonnes : Boulogne, Lorient, Le Guilvinec et Concarneau. En 2013, six quartiers accèdent à une production supérieure à 10000 tonnes, mais seul le quartier du Guilvinec se place juste au-delà de 30000 tonnes (figure 2). La baudroie ou lotte (Lophius piscatorius) et la langoustine (Nephrops norvegicus) constituent deux espèces emblématiques au sein de ce quartier maritime.

Figure 2 Evolution des productions en tonnes et des prix (courbes) en euros constants base 2013 à l'échelle nationale $(a)$ et dans le quartier maritime du Guilvinec $(b)$

(a)

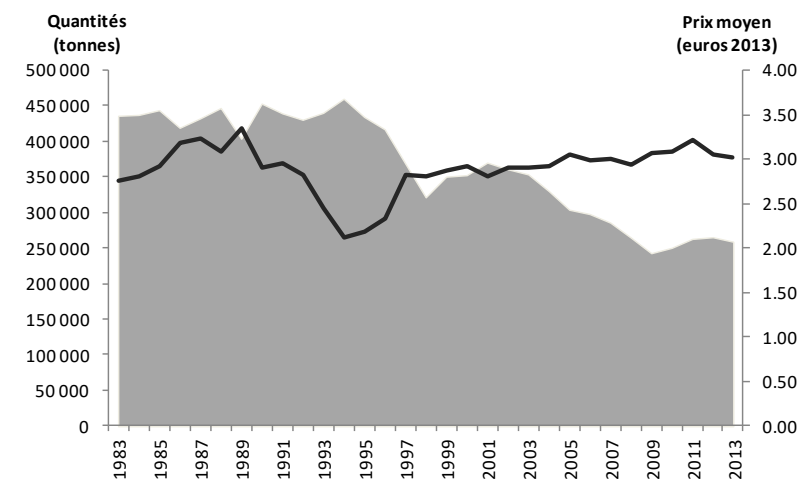

(b)

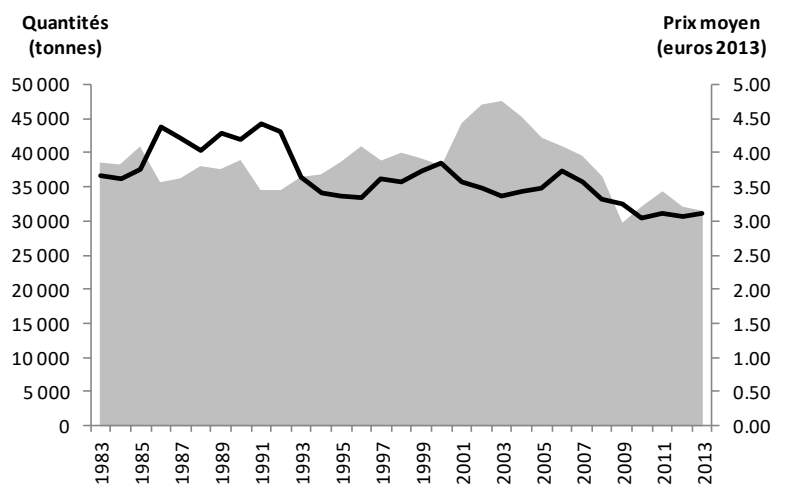

Source : LE FLOC'H et WILSON, 2017 
La production des pêches maritimes (fig. $2 a$ ) est commercialisée, en première vente, dans un réseau de 39 halles à marée. Les 20 premières, du Guilvinec au Croisic (fig. 3), représentent 83\% de la production en valeur pour l'année 2014, soit 500 millions d'euros (FrANCEAGRIMER, 2014).

La commune du Guilvinec est le territoire le plus marqué par la présence des activités de la sphère productive ${ }^{2}$ liés directement et indirectement aux activités de la filière pêche. D'autres activités, sans lien direct avec la pêche, contribuent aux emplois de la sphère productive. Les emplois inscrits dans la sphère productive (61\% du total des emplois recensés sur la commune) dépassent ceux de la sphère présentielle (39\%), indiquant que l'essentiel des postes de travail se situe dans l'activité d'exportation des produits, hors des limites de la commune. La partition des emplois selon l'INSEE répond à une logique de spatialisation des activités économiques.

Figure 3 Répartition des emplois de la sphère productive et de la sphère présentielle dans les communes détenant une halle à marée en 2014

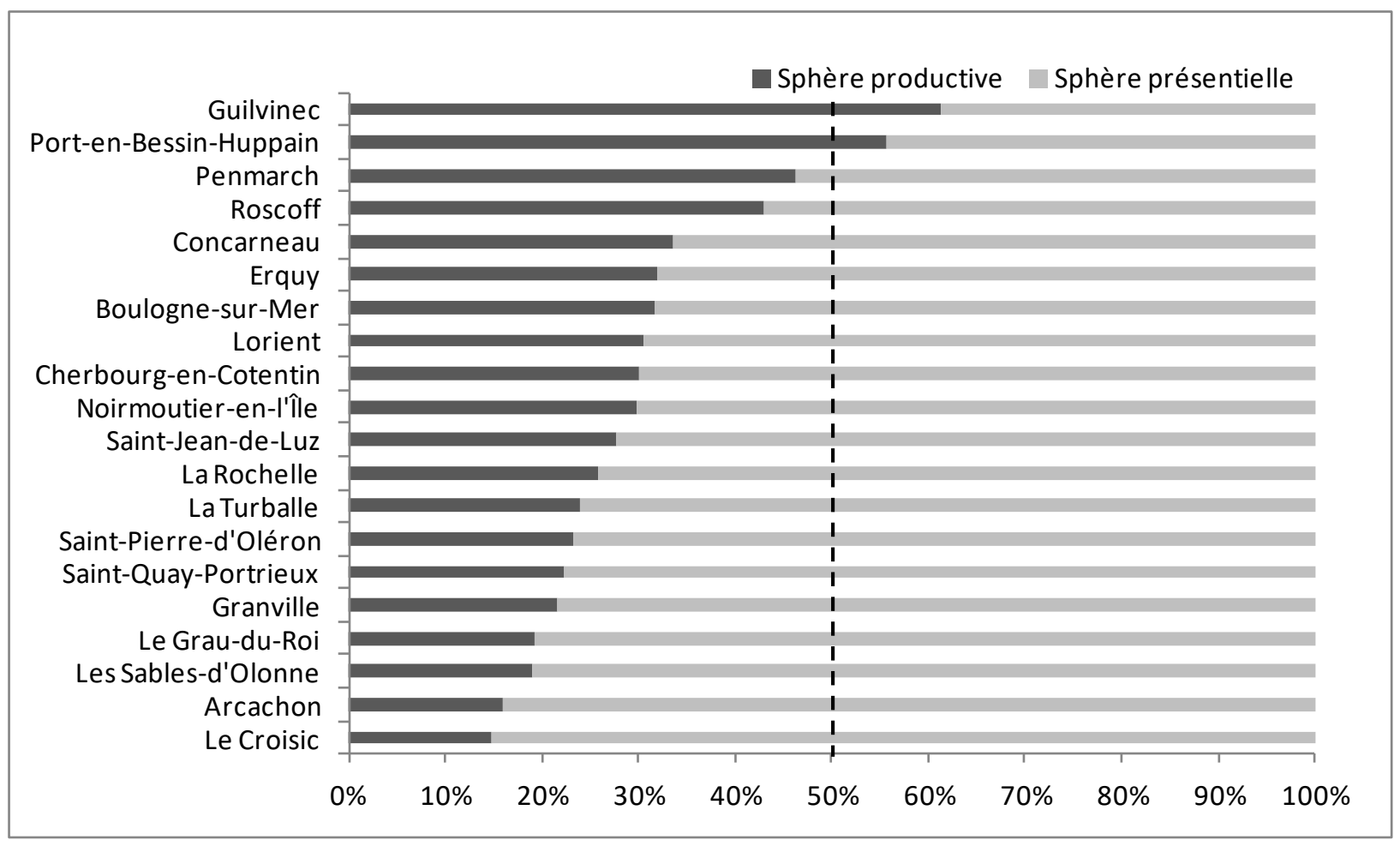

Source : INSEE

\footnotetext{
${ }^{2}$ Selon l'INSEE, « les activités présentielles sont les activités mises en œuvre localement pour la production de biens et de services visant la satisfaction des besoins de personnes présentes dans la zone, qu'elles soient résidentes ou touristes. Les activités productives sont déterminées par différence. Il s'agit des activités qui produisent des biens majoritairement consommés hors de la zone et des activités de services tournées principalement vers les entreprises correspondantes ».
} 
Le port de pêche du Guilvinec occupe la première place des 39 halles à marée, suivi de Lorient et Boulogne-sur-Mer où la structure des emplois se rapproche des métropoles régionales. Même dans les communes de taille proche à celle du Guilvinec (Erquy, Saint-Pierre d'Oléron, SaintQuay-Portrieux ou Granville), les emplois de la sphère présentielle dépassent largement ceux de la sphère productive avec un rapport inversé comparativement au premier port de pêche.

Une comptabilité des postes salariés inscrits dans la sphère productive (production exportée hors de la zone) plus forte que celle des postes de la sphère présentielle (production non exportée) témoigne de la création d'un système productif local -SPL- ouvert (INSEE, 2015). La littérature consacrée au lien entre territoire et phénomène d'agglomération industrielle propose un concept spécifique à l'agroalimentaire, le SYAL ou Système agroalimentaire localisé, qui convient également à l'industrie des pêches maritimes (REQUIER-DESJARDINS, 2010). Ce concept semble même plus adapté au modèle du panier de biens, décrit ci-dessous, que celui de SPL. Une déclinaison aux produits de la pêche serait le système halio-alimentaire localisé (GUILLOTREAU, 1992).

La question de la base territoriale sur laquelle son fixées des activités de valorisation des ressources locales est en soi une problématique de recherche pour identifier l'échelle spatiale pertinente, par exemple pour adapter des politiques publiques d'aménagement. L'analyse menée conjointement sur les pêches maritimes et le secteur du tourisme impose de recenser les différents échelons. On relève deux unités spatiales dans la gestion à terre des flottilles de pêche, la commune dans laquelle se situe l'infrastructure portuaire, et le quartier maritime regroupant des communes portuaires proches. La commune du Guilvinec est donc un premier échelon territorial. Le quartier maritime du Guilvinec étend les limites à toutes les communes portuaires proches (Loctudy, Lesconil, Le Guilvinec et Saint-Guénolé). Le tourisme littoral déborde les limites des commues dédiées à la pêche. Notamment, le comité régional du tourisme en Bretagne définit dix grandes destinations, dont le territoire de Cornouaille qui regroupe quatre quartiers maritimes (du Nord au Sud: Douarnenez, Audierne, Le Guilvinec, Concarneau). La base territoriale des attractions touristiques liées aux pêches maritimes dans notre étude de cas peut donc être retenue sur trois échelons imbriqués (commune Le Guilvinec, quartier maritime Le Guilvinec, territoire de Cornouaille). La question de la base territoriale et de son attractivité s'est imposée au début des années 1980 en posant le postulat d'une différenciation entre un espace appropriable et un territoire qui ne l'est pas (LEON et SAUVIN, 2010).

Le complexe halieutique du Guilvinec abrite toutes les activités de support au métier de marin pêcheur : construction et réparation navale, approvisionnement, manutention et première vente, transformation et transport, poissonnerie. Le choix d'implanter un site de découverte des métiers de la pêche destiné à la population résidente et touristique au sein même du complexe industriel démontre le souhait des partenaires publics et privés du territoire d'exploiter les interdépendances entre pêche et tourisme. Or, la coordination des acteurs du territoire est l'une des clés du succès du modèle d'un panier de biens (HIRCZAK et al., 2008).

A la fin des années 1990, la commune du Guilvinec prend conscience d'une perte d'attractivité de son industrie de base par la diminution des navires de pêche. Pourtant le port de pêche attire plus de 150000 visiteurs chaque année sur les quais pour assister au débarquement du poisson et des crustacés (ALBAN, 1998). La gestion d'un flux touristique important répond autant à des enjeux 
de sécurité au moment de l'organisation de la vente du soir qu'à une volonté d'exploitation commerciale de la part des acteurs publics et privés du territoire.

Afin de créer des synergies entre les deux secteurs et dynamiser son économie locale, la commune décide de capter ce flux en proposant une nouvelle infrastructure touristique nommée Haliotika, créée en 2000. Cette "Cité de la pêche » informe les visiteurs sur le métier de marin pêcheur et plus largement sur l'ensemble des professions de la filière pêche. La mise en valeur d'un patrimoine local (matériel et immatériel), et en particulier du patrimoine halieutique, contribue à l'image de la commune et de ses environs et à son économie en renforçant son attractivité touristique (KOSIANSKI et MONINO, 2005 ; PERON et MARIE, 2010).

Les objectifs de cet outil de valorisation des ressources territoriales (PECQUEUR, 2001) sont tournés vers une meilleure connaissance des métiers de la pêche auprès du grand public, afin d'encourager la consommation des produits issus de la pêche locale et mobiliser les acteurs du territoire autour du centre de découverte.

Sous régie municipale au cours des premières années d'exploitation, le centre touristique devient une société d'économie mixte en 2007 associant à la communauté de communes des partenaires privés représentant des entreprises de transformation des produits de la mer. L'équipement reçoit un soutien financier des collectivités territoriales pour l'investissement mais assure depuis 2012 son autonomie de fonctionnement.

Le chiffre d'affaires du centre de découverte a progressé de $57 \%$ en euros courants entre 2009 et 2016, atteignant $402 \mathrm{k} €$ (figure 4). Rapporté au chiffre d'affaires de la baudroie, 30 millions d'euros en 2015, ou de la langoustine, 18 millions d'euros (IFREMER, 2017), la contribution économique des visiteurs par le paiement des entrées reste marginale. La fréquentation, inférieure à 40000 visites en 2009 et 2010, franchit ce seuil dès 2011 et se situe autour de 50000 entrées depuis 2014. D'autres retombées économiques, plus diffuses, renforcent les interdépendances entre pêche et tourisme à l'échelle d'un territoire dont le rayonnement attractif n'est pas clairement identifié. On relève en effet trois espaces imbriqués qui peuvent servir de support aux politiques territoriales, la commune du Guilvinec, le quartier maritime du Guilvinec associant les communes portuaires de proximité (Saint-Guénolé, Lesconil et Loctudy), et le territoire de Cornouaille (Est et Ouest de Quimper). On doit en effet se poser la question du rayonnement territorial du site (CHARLES et THOUEMENT, 2007).

La clientèle d'Haliotika se compose de trois principaux segments : les visiteurs individuels, les groupes et les scolaires (fig. 4). La population touristique, confondue avec la population locale, se situe dans les deux premiers segments, les visiteurs individuels (60 à 70\%) et des groupes (15 à 20\%). On retient la définition de l'Organisation Mondiale du Tourisme séparant les visiteurs en deux catégories, les touristes qui passent une nuit au moins hors de leur domicile et les excursionnistes sans nuitée extérieure à leur résidence. 
Figure 4 Evolution de la fréquentation (individuels, groupes et scolaires) et du chiffre d'affaires (courbe)

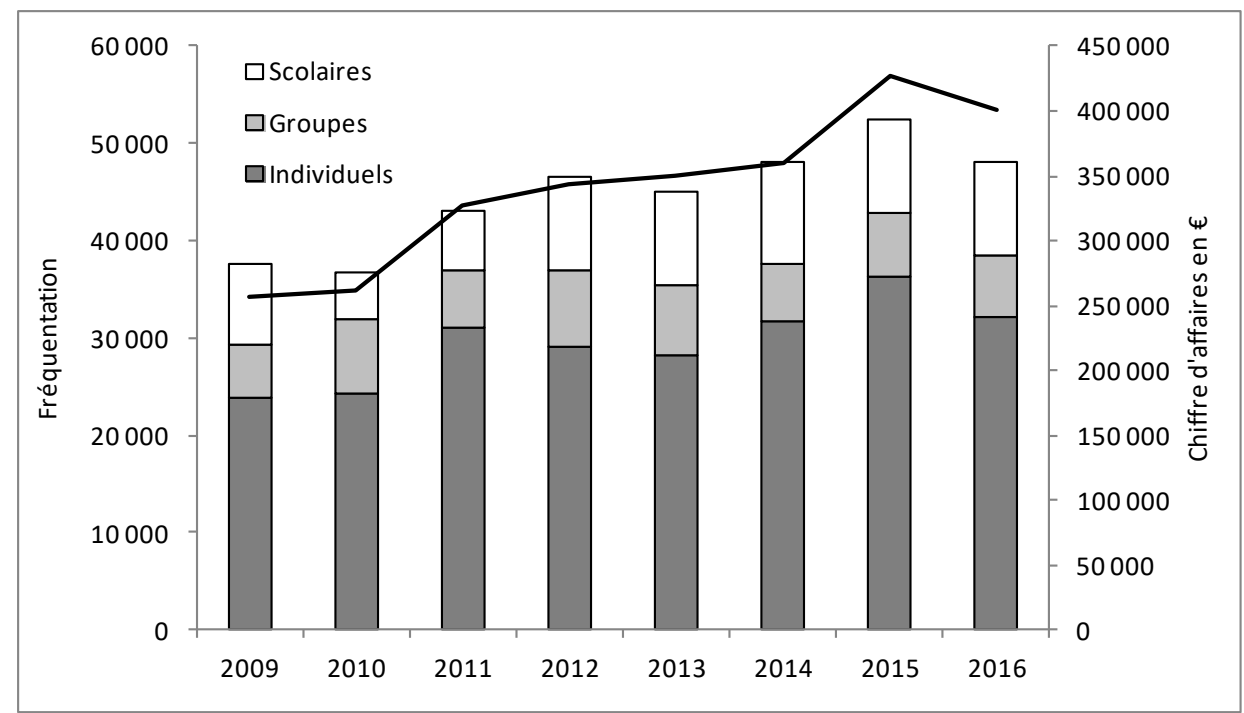

Source : Elaboration propre d'après Haliotika

Haliotika est décrit dans cette recherche comme un panier de biens en maîtrisant une diversité de biens en un même lieu, deux caractéristiques reconnus à cette représentation conceptuelle. Les visiteurs individuels choisissent eux-mêmes le type de visite, comprenant plus de 14 choix, dont la visite du centre, la visite du centre et celle de la criée, une journée immersion avec dégustation des produits de la mer, une découverte de l'écosystème côtier, un embarquement sur un chalutier $^{3}$... Depuis sa création, le centre a énormément élargi son offre, avec notamment des ateliers à destination des enfants, des ateliers culinaires,etc.

Quasiment chaque bien pris individuellement peut être consommé en d'autres lieux, sans rapport avec le centre Haliotika. D'autres sites consacrés à la mer, et pas nécessairement sur le littoral, offrent une possibilité de consommation d'un seul bien (aquariums, musée de la pêche, compagnies maritimes, cours de cuisine) et sans nécessairement avec un ancrage territorial. Le modèle du panier de biens, décrit à la prochaine section, propose un support théorique pertinent dans l'analyse de l'attractivité territoriale du centre de découverte des métiers de la pêche. Les deux caractères qu'emprunte le modèle du panier de biens aux autres modèles de consommation sont « le caractère composite de l'offre et...le caractère situé de l'offre » (PECQUEUR, 2001).

La démarche empirique, décrite dans la section 6, soutenue par l'approche du panier de biens s'inscrit dans un cadre conceptuel large. La problématique du caractère territorial des attractions touristiques liées aux pêches maritimes est multidimensionnelle car elle dépasse le cadre classique d'une demande sans attache territoriale. Les concepts abordés sont le panier de biens (PECQUEUR, 2001), la base territoriale pertinente comme facteur d'attractivité (ZIMMERMANN, 2005), les

\footnotetext{
${ }^{3}$ D'autres ports de pêche organisent, par les offices de tourisme, la visite de la halle à marée ou criée sans offrir un choix plus large. Les visiteurs peuvent donc opter sur le même territoire de Cornouaille pour la seule visite de la halle à marée (Audierne par exemple).
} 
motivations multiples des visiteurs en séjour touristique (LEIPER, 1990), et la question de la rente de qualité territoriale (FONNER and SYLVIA, 2015). L'article propose un examen détaillé des deux premiers concepts, en revisitant la typologie des modèles de paniers de biens et en cernant, sur la base des résultats d'enquêtes, l'échelle territoriale pertinente. La discussion reprend l'ensemble du cadre conceptuel, notamment la question de la rente de qualité territoriale.

\section{$-5-$}

\section{Le modèle du panier de biens revisité}

Le modèle du panier de biens (Pecqueur, 2001; Mollard, 2001) a fait l'objet d'un article de synthèse sur les travaux empiriques engagés au cours des années 2000 (HIRCZAK et al., 2008). On trouve peu de références récentes dans la littérature francophone sur le modèle du panier de biens, si ce n'est un rapide détour sans éléments théoriques (BAZIN-BENOÎT et al., 2014), ou un appel plus prononcé sur le concept de rente de qualité territoriale mais sans illustration nouvelle d'un panier de biens (KREZIAK et al., 2012 ; ARNAL, 2012).

D'autres recherches ont depuis creusé un sillon proche, combinant les concepts d'ancrage territorial, d'identité et de qualité des produits (CANADA et MUCHNIK, 2011). Des approches renouvelées sont en état d'émergence (économie circulaire, économie des fonctionnalités) ou en développement (écologie industrielle) pour cerner les interactions entre territoire, identité et qualité des produits (MAILLEFERT et ROBERT, 2017). Cependant, le modèle du panier de biens garde toute sa pertinence pour expliquer le comportement du consommateur dans un contexte récréatif (HOLBROOK and HiRSCHMAN, 1982).

Un rappel des fondements, critères et typologie du modèle de panier de biens permet de mieux comprendre sa pertinence. Le modèle est scientifiquement fondé car il s'inspire de l'approche renouvelée de la théorie du consommateur par LANCASTER (1966) révélant l'utilité procurée non par le bien lui-même mais par ses attributs ou caractéristiques. Les axiomes de l'approche standard (préférence complète, préférences transitives, non-saturation et convexité des préférences) laissent place, sans être totalement ignorées, à trois hypothèses (PECQUEUR, 2001) dont l'une ouvre la voie au modèle du panier de biens, c'est à dire une combinaison de biens distincts.

Les critères retenus du modèle du panier de biens (i) posent le consommateur comme le libre arbitre dans la composition de son panier, (ii) limitent le choix aux services et produits localisés sur le même territoire, (iii) reconnaissent dans les biens sélectionnés par le consommateur un effet qualité. Les cas d'étude recensés au cours des années 2000 (HIRCZAK et al., 2008) placent le modèle du panier de biens dans un contexte récréatif avec une demande locale et une demande touristique, révélant un consentement à payer plus fort pour des biens associés, produits localement et dans une démarche de qualité. Ce qui distingue ce modèle de la théorie lancastérienne sur les attributs ou les travaux de biens associés avec un prix unique mais fabriqués en divers lieux (BoJAMIC and Calantone, 1990), ce sont les valeurs non incorporées directement au panier, qui relèvent de la mémoire d'un lieu ou d'un imaginaire (GAGNON, 2007). Ce consentement à payer plus élevé pour un ensemble de biens et services, observé dans le comportement d'une clientèle touristique, crée une rente de qualité territoriale dès que les produits exportés, hors du territoire de production, suscitent un acte d'achat à un prix supérieur à des produits ou services concurrents mais sans 
attache territoriale spécifique. Les pêches maritimes offrent de nombreux exemples, par des espèces halieutiques symboliques (la morue, la coquille Saint-Jacques, la sardine....), mais rarement mis en valeur par une exploitation commerciale ciblant une clientèle touristique. Par exemple, la pêcherie de homard en Amérique du Nord dans l'Etat du Maine est célébrée depuis plus de 70 ans par un évènement festivalier de grande ampleur. Le flux touristique de masse est perçu dans un contexte proche du panier de biens, avec la fréquentation des villages côtiers, des plages et la restauration des produits de la mer issus du même territoire (DANIEL et al., 2008). Les aménités environnementales et patrimoniales comptent tout autant que la consommation de l'espèce emblématique (LESUEUR et al., 2014).

L'application du modèle du panier de biens au cas des pêches maritimes impose de revisiter la typologie élaborée à l'aune des cas d'étude situés en milieu pastoral ou agricole. HIRCZAK et al. (2008) proposent quatre variantes du modèle repris dans le tableau 1 en désignant la caractéristique principale, la problématique et la solution envisageable pour se rapprocher d'un véritable panier de biens (choix du consommateur, ancrage territorial des biens, démarche de qualité).

Tableau 1 Typologie revisitée des modèles du panier de biens

\begin{tabular}{|c|c|c|c|}
\hline & Caractéristique & Problématique & Solution \\
\hline Modèle du panier de biens & $\begin{array}{l}\text { Marginalisation } \mathrm{du} \\
\text { territoire par la } \\
\text { modernisation }\end{array}$ & $\begin{array}{l}\text { Question de pérennité du } \\
\text { modèle }\end{array}$ & Création de parcs \\
\hline Modèle du panier hybride & $\begin{array}{l}\text { Coexistence de } \\
\text { produits génériques } \\
\text { et de produits de } \\
\text { qualité territoriale }\end{array}$ & $\begin{array}{l}\text { Tradition ou modernité du } \\
\text { processus de production } \\
\text { territorial }\end{array}$ & $\begin{array}{l}\text { Améliorer la } \\
\text { coordination entre les } \\
\text { acteurs du territoire }\end{array}$ \\
\hline Le modèle centralisé & $\begin{array}{lr}\text { Stratégies } & \text { des } \\
\text { institutions } & \text { supra- } \\
\text { territoriales } & \end{array}$ & $\begin{array}{l}\text { Le tourisme comme } \\
\text { produit d'appel }\end{array}$ & $\begin{array}{l}\text { Vers une } \\
\text { décentralisation par } \\
\text { une implication plus } \\
\text { forte des acteurs } \\
\text { locaux }\end{array}$ \\
\hline $\begin{array}{l}\text { Le modèle d'assortiments } \\
\text { juxtaposés }\end{array}$ & $\begin{array}{l}\text { Offre variée mais } \\
\text { une faible référence } \\
\text { au lieu d'origine }\end{array}$ & $\begin{array}{l}\text { Logique de conflits entre } \\
\text { les acteurs privés }\end{array}$ & $\begin{array}{l}\text { Nécessité d'une } \\
\text { coordination entre } \\
\text { acteurs publics et } \\
\text { privés }\end{array}$ \\
\hline
\end{tabular}

Source : d'après HIRCZAK et al., 2008

Tirée de l'expérience de cas d'étude, cette typologie n'impose pas une seule trajectoire de croissance pour les territoires ruraux ou littoraux. Le cas d'étude présenté dans cet article emprunte certainement aux quatre variantes. En effet, le centre de découverte Haliotika est situé sur un 
territoire tenté par la tradition et la modernité, c'est-à-dire une pêche côtière proposant des produits frais de la journée et une pêche du large beaucoup plus intensive en énergie (modèle du panier hybride). Toutefois, la création Parc naturel marin d'Iroise en 2007, premier parc naturel marin en France, est une opportunité bien saisie par certains pêcheurs professionnels pour valoriser les productions tirées des pêcheries situées à l'intérieur du parc (LEONARDI et al., 2010). C'est un élément présent comme solution possible à la pérennité du modèle du panier de biens (modèle du panier de biens proprement dit). On retrouve également des éléments propres aux modèles 3 et 4 dans l'exemple d'attractions touristiques liées aux pêches maritimes, tels que des stratégies supranationales (politiques de reconversion des pêches vers le tourisme) et une offre de produits de la mer peu lisible pour le consommateur (appellation d'origine Atlantique Nord-Est du poisson sur étalage).

Les éléments quantitatifs apportés dans cette recherche relèvent d'une approche classique d'enquête auprès d'une population touristique. C'est vers une réflexion inversée à la théorie de la base que sont valorisés les résultats de l'enquête, (i) en cherchant à identifier les contours du territoire de résidence de la clientèle touristique au cours de leur séjour puis (ii) en estimant le montant des dépenses des visiteurs. La visite du centre de découverte Haliotika offre un ensemble de biens complémentaires et dépendants, et peut déclencher chez le visiteur un acte d'achat plus fréquent de produits de la mer au cours du séjour touristique.

\section{$-6-$}

\section{Méthode et données}

La méthodologie de collecte de données qualitatives et quantitatives par enquête mise en œuvre dans le cadre de cet article répond à un double objectif : (i) délimiter le territoire sur lequel s'exerce une attractivité des activités de pêche et du centre de découverte, et (ii) fournir une estimation des dépenses engagées par les visiteurs au cours de leur séjour touristique sur ce même territoire.

La localisation des visiteurs d'après leur résidence principale et leur lieu de séjour touristique apporte de nouveaux éléments de connaissance sur le caractère territorial du site d'attraction touristique des pêches maritimes. Une première enquête en face à face, conduite à l'issue immédiate de la visite du centre, aborde les questions de localisation et de perception. Une seconde enquête produite deux semaines après la visite, par couriel, cherche à estimer les dépenses monétaires effectuées pendant le séjour.

\subsection{Enquête sur la perception et la localisation}

La première phase de l'enquête s'est déroulée en mai et juin 2017 à la sortie du centre de découverte Haliotika, partenaire de l'étude menée dans le cadre d'un stage de Master 2 (POCHEAU, 2017). Une équipe de quatre enquêteurs a organisé les entretiens en face à face. Outre le profil socio-économique des visiteurs, le premier questionnaire aborde quatre aspects, à savoir: la notoriété du centre de découverte Haliotika, la consommation des produits de la mer, la perception du métier de marin pêcheur et la localisation (résidence principale et lieu du séjour touristique).

L'échantillon comprend 168 individus enquêtés individuellement (tableau 2), soit 3\% des entrées relevées sur le mois de juin. 5000 à 6000 visiteurs mensuels sont comptabilisés d'avril à 
juin. Le chiffre s'élève à près de 8000 en juillet et atteint un plafond de 11000 à 12000 en août. Une partie des enquêtés sont excursionnistes, sans déclaration d'une nuitée hors de leur domicile.

Tableau 2 Echantillon de la première enquête

\begin{tabular}{|c|c|c|c|c|c|c|}
\hline & Effectif & $\begin{array}{c}\text { Age } \\
\text { moyen }\end{array}$ & Retraités & Actifs & $\begin{array}{c}\text { Département } \\
\text { littoral }\end{array}$ & $\begin{array}{c}\text { Département } \\
\text { non littoral }\end{array}$ \\
\hline Femme & 81 & 61 & 61 & 32 & 42 & 51 \\
Homme & 87 & 58 & 40 & 35 & 39 & 36 \\
\hline Total & $\mathbf{1 6 8}$ & $\mathbf{5 9}$ & $\mathbf{1 0 1}$ & $\mathbf{6 7}$ & $\mathbf{8 1}$ & $\mathbf{8 7}$ \\
\hline
\end{tabular}

Source : POCHEAU, 2017

La structure de l'échantillon indique une parité entre les femmes et les hommes et un âge moyen de 59 ans. Une majorité de retraités fréquente le centre de découverte sur le mois de juin. On remarque toutefois que l'écart entre retraités et actifs est peu important chez les hommes. Les variables «département littoral » et «département non littoral » indiquent la distance géographique entre le lieu de résidence principale et le littoral. Les individus déclarant une résidence principale dans un département littoral sont considérés comme proches de la mer (AlpesMaritimes, Calvados, Charente-Maritime, Côte d'Armor, Eure, Finistère, Ille-et-Vilaine, LoireAtlantique, Manche, Morbihan, Nord, Pyrénées-Atlantiques, Seine-Maritime, Somme, Vendée) auxquels s'ajoutent des visiteurs originaires d'Espagne, du Royaume-Uni et de Norvège. Un peu moins de la moitié de l'échantillon, 81 individus, habite dans un département littoral, ce qui ne signifie pas nécessairement une forte proximité géographique avec la mer. Une courte majorité des visiteurs rencontrés réside dans des départements sans espace côtier.

Une majorité forte de clients préfère la visite libre (64\%) à une visite guidée (36\%). En revanche, la combinaison de la découverte du centre (libre ou guidée) avec la visite de la criée aux heures des ventes de la pêche du large aux aurores ou de celle de la pêche côtière en fin de journée est un choix fréquent (73\% de l'échantillon). Un seul enquêté a également ajouté l'atelier cuisine. Aucune réponse n'a été relevée pour la sortie en mer. Toutefois, 150 embarquements ont été organisés sur l'année 2017.

Le panier de biens est réduit, dans les choix opérés par les visiteurs du mois de juin, à deux types de consommation sur l'ensemble des services offerts. C'est ici une limite importante de l'étude, centrée sur la clientèle d'avant saison estivale.

A l'issue de l'entretien, les enquêteurs ont proposé d'adresser un second questionnaire dans les deux semaines par contact électronique. 57 individus ont répondu au second questionnaire portant sur les dépenses en saisissant directement leurs réponses sur une interface du logiciel Sphinx. 


\subsection{Enquête sur les dépenses au cours du séjour}

Le second questionnaire débute avec des questions sur les habitudes alimentaires et la fréquence d'achat de produits de la mer au cours du séjour. Le profil de l'enquêté s'appuie sur les caractéristiques socio-démographiques et la durée du séjour. Le point central de cette seconde enquête détaille les postes de dépenses d'hébergement, de restauration, les dépenses de loisir dont le coût de la visite à Haliotika, l'achat de produits de la mer et une catégorie divers achats (tels que les souvenirs du séjour). Un effet possible du panier de biens est de déclencher un acte d'achat supérieur après la visite du centre de découverte, notamment sur les produits de la mer.

L'estimation globale des dépenses touristiques est pondérée par la taille du ménage. Dans les travaux de l'INSEE des comptes satellites du tourisme, ces postes de dépenses effectuées sur les territoires visités représentent à l'échelle nationale 71\% du budget « vacances » des ménages. 19\% représentent les dépenses de transport (carburants, péages, location de véhicule et services de transport non urbains). Les achats des touristes sur site atteignent près de $80 \%$ en Bretagne, comme en Languedoc-Roussillon et Poitou-Charentes (INSEE, 2014).

L'échantillon se compose de 57 individus, dont 32 visiteurs dans la catégorie visite individuelle et 25 visites au sein d'un groupe (tableau 3 ).

Tableau 3 Echantillon de la seconde enquête, moyenne et écart-type

\begin{tabular}{|l|c|c|c|}
\hline Visiteurs & Effectif & $\begin{array}{c}\text { Taille du } \\
\text { ménage }\end{array}$ & $\begin{array}{c}\text { Durée du séjour en } \\
\text { jour }\end{array}$ \\
\hline Individuels & 32 & $\begin{array}{c}2.9 \\
(1.7)\end{array}$ & $\begin{array}{c}3.3 \\
(4.7)\end{array}$ \\
\hline Groupes & 25 & 1.8 & 0.7 \\
& & $(0.7)$ & $(0.2)$ \\
\hline Total & 57 & 2.2 & 2.1 \\
& & $(1.5)$ & $(3.7)$ \\
\hline
\end{tabular}

Source : POCHEAU, 2017

L'estimation des retombées économiques directes distingue les visiteurs individuels des visites en groupe avec une description méthodologique inspirée d'un exercice mené à partir de l'attractivité des aires marines protégées (RONCIN et al., 2008). Les répondants à la seconde enquête sont qualifiés de touristes, déclarant au moins une nuitée hors de leur lieu de résidence principale.

Les dépenses globales moyennes des visiteurs au cours de leur séjour cumulent les quatre postes d'hébergement, de restauration, d'achats de produits de la mer et autres loisirs, en séparant les visiteurs inscrits en individuels des visiteurs au sein de groupes (figure 5). La dépense moyenne par personne, calculée selon la taille des ménages, est extrapolée à la population de visiteurs déclarés par Haliotika. Cette méthode présente trois limites : (i) une partie des visiteurs n'est pas 
en séjour touristique, (ii) les visiteurs en séjour touristique ne résident pas nécessairement sur la commune du Guilvinec, (iii) une minorité des visiteurs fait le déplacement spécifiquement pour visiter Haliotika. Ainsi, il n'est pas possible d'affirmer que les retombées économiques estimées sont le seul fait de l'existence d'Haliotika. LEIPER (1990) classe les attractions touristiques en trois catégories : celles dites "primaires" qui motivent la venue du touriste, celles dites "secondaires" qui sont également connues de lui avant le départ, et les attractions dites "tertiaires" qui sont découvertes une fois sur place et participent à l'agrément du séjour. Les attractions touristiques localisées sur un même territoire ou des territoires proches soulèvent une problématique plus large que celle abordée dans cet article, celle des multi-motivations des touristes. L'analyse de ce sujet est fréquent lorsqu'on estime les retombées économiques d'une manifestation sportive ou culturelle, ou d'une aire protégée (RONCIN et al., 2008 ; CARLSEN and WoOD, 2004). Dans ce cas, il est nécessaire d'utiliser un facteur d'attribution des dépenses, estimé à partir des enquêtes. Le problème est le même que dans le cas de déplacement multi-destination, multi-motivation pour appliquer la méthode des coûts de transport (MARTínEZ-EsPIÑEIRA and AMOAKO-TUFFOUR, 2009; VOLTAIRE et al., 2017). Les motivations de la clientèle se concentrent sur la visite de la criée combinée à la visite du centre. Cette option apparaît comme un facteur fortement attractif puisque $73 \%$ des visiteurs ont sélectionné ce panier.

Toutefois, dans le cas qui nous intéresse, on peut aisément supposer que le patrimoine maritime et halieutique du port du Guilvinec constitue une motivation importante de cette destination touristique «connue », et que le centre de découverte Haliotika est, en quelque sorte, l'infrastructure concrète qui renforce l'interdépendance entre pêche et tourisme à l'échelle de ce territoire.

Figure 5 Méthode d'estimation des retombées économiques générées par les visiteurs 


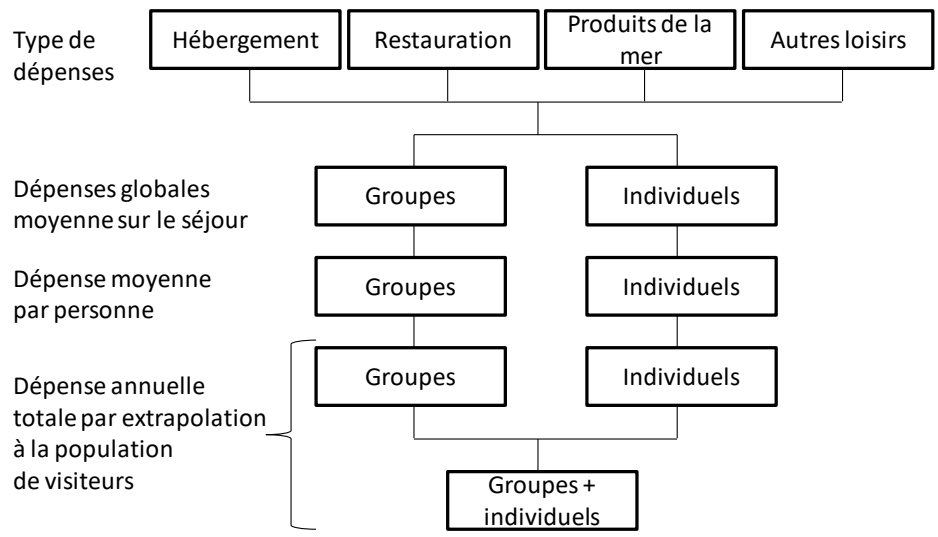

Source : élaboration propre, d'après RONCIN et al., 2008

$-7-$

Résultats

La littérature sur les choix de visite touristique décrit une hiérarchie avec un premier élément ( «nucleus ») des sites connus et attractifs, un second élément de sites connus mais non désirés au moment du choix, un troisième éléments sur les sites inconnus avant le voyage (HolBROOK and HIRSCHMAN, 1982 ; LEIPER, 1990). On peut aisément supposer que pour l'essentiel des visiteurs, le centre de découverte Haliotika fait partie de la 2e ou 3e catégorie. Sur la première enquête, $47 \%$ des 168 visiteurs se sont rendus à Haliotika par curiosité, 33\% pour un intérêt sur la problématique des pêches maritimes et $20 \%$ sans motif particulier. $43 \%$ des touristes interrogés ont également visité un autre centre lié à la mer sur la même période de vacances (musée de la pêche à Concarneau ou Douarnenez, Océanopolis à Brest). Pour mieux cerner l'origine géographique des touristes, le premier résultat précise la localisation des visiteurs par leur lieu de résidence principale et le lieu de leur séjour de vacance. Le second résultat propose une estimation des dépenses sur le territoire d'implantation du centre de découverte de la pêche.

\subsection{Localisation des visiteurs}

La localisation du lieu de résidence principale des visiteurs s'appuie sur l'ensemble de l'échantillon constitué de 168 répondants à l'enquête. 150 des enquêtés résident à titre principal dans un département français et 18 déclarent une résidence à l'étranger (Allemagne, Belgique, Norvège, République Tchèque, Royaume-Uni, Singapour, Suisse). Les bretons représentent 29\% de l'échantillon, dont $20 \%$ du département du Finistère.

La figure 6 fournit une première indication sur l'origine géographique des visiteurs en début de saison estivale. Sur la base des individus résidant en France, le centre de découverte présente un intérêt pour une population souvent éloignée du littoral (Centre Est). On observe cependant une plus forte concentration dans les régions du Grand Ouest, d'Ile de France et du Nord. 
Figure 6 Lieu de résidence principale des visiteurs au mois de juin
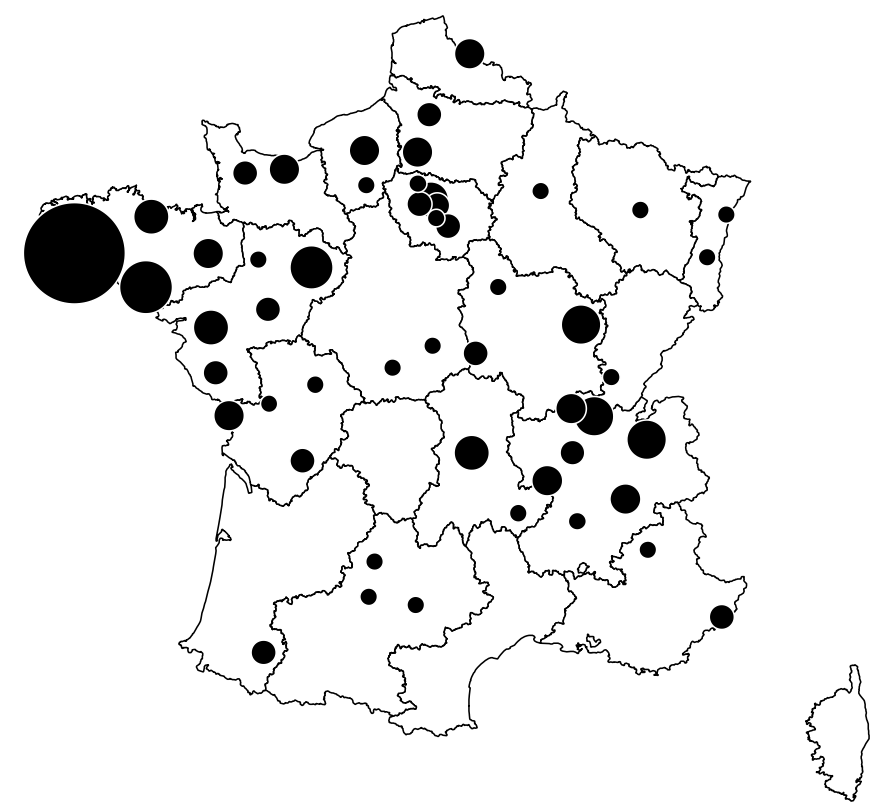

SOUrCe : POCHEAU, 2017

Plus des $3 / 4$ des répondants déclarent un lieu de séjour distinct de leur lieu de résidence principale (figure 7). Le choix de visite du centre de découverte de la pêche s'inscrit par conséquent dans le cadre d'un séjour touristique avec au moins une nuitée en dehors de l'habitation principale. Ce lieu d'hébergement touristique est à proximité du port de pêche du Guilvinec. 90\% se situent au sein du territoire de Cornouaille, un des dix territoires touristiques identifiés à l'échelle de la région Bretagne. Les quatre ports du quartier maritime du Guilvinec (Saint-Guénolé, Guilvinec, Lesconil et Loctudy) sont à l'Ouest de la Cornouaille. L'attractivité du centre de découverte n'est cependant pas davantage prononcée au sein même des limites du quartier maritime du Guilvinec. Les visiteurs du centre de découverte sont aussi nombreux à l'Est (55) qu'à l'Ouest du territoire de Cornouaille (59). Le choix du territoire pertinent dans l'étude de l'attractivité du centre Haliotika penche par conséquent sur l'ensemble de la Cornouaille, plutôt que le quartier maritime ou la commune du Guilvinec. 
Figure 7 Lieu de résidence touristique des visiteurs

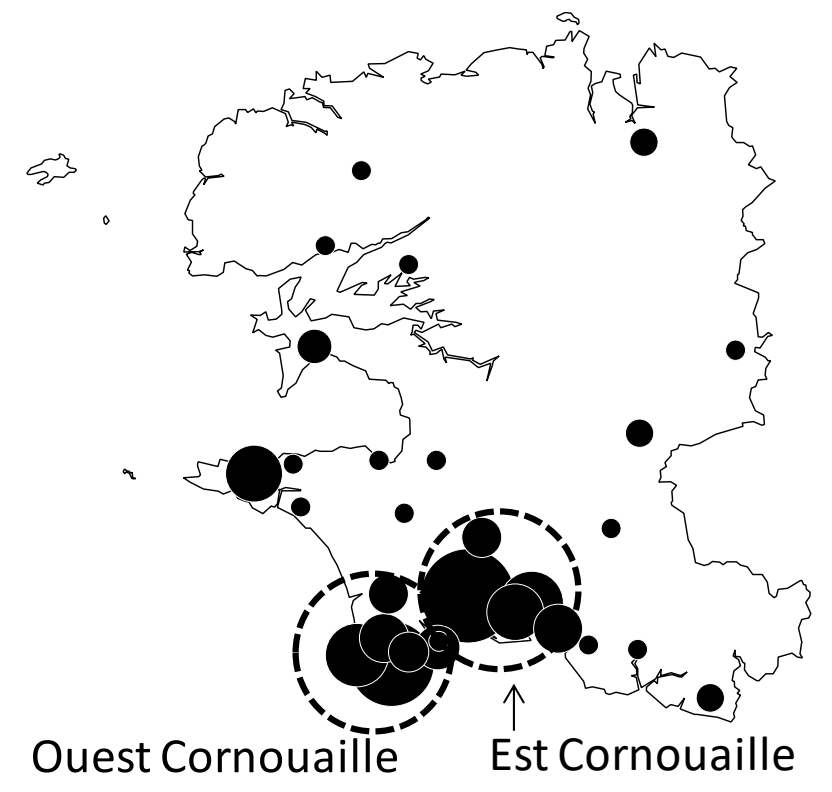

SOURCe : POCHEAU, 2017

\subsection{Les dépenses des visiteurs}

Les dépenses des touristes attirés par le centre de découverte comprennent l'hébergement, la restauration, l'achat de produits de la mer, les entrées à Haliotika et autres dépenses. Sur les 57 individus de la seconde enquête, 36 ont déclaré des dépenses sur la commune du Guilvinec, en plus du droit d'entrée au centre de découverte (tableau 4). La taille de l'échantillon des répondants sur l'aspect des dépenses sur la commune est faible mais fournit des estimations précises.

Tableau 4 Les dépenses des visiteurs en euro, moyenne et écart-type

\begin{tabular}{|c|c|c|c|c|c|c|}
\hline & \multicolumn{1}{|c|}{$\begin{array}{c}\text { Héber- } \\
\text { gement }\end{array}$} & $\begin{array}{c}\text { Restau- } \\
\text { ration }\end{array}$ & $\begin{array}{c}\text { Produits } \\
\text { de la mer }\end{array}$ & $\begin{array}{c}\text { Autres } \\
\text { loisir }\end{array}$ & $\begin{array}{c}\text { Dépenses } \\
\text { totales }\end{array}$ & $\begin{array}{c}\text { Dépenses } \\
\text { par personne }\end{array}$ \\
\hline Individuels & 256.2 & 92.4 & 69.4 & 101.4 & 519.4 & 179.1 \\
\hline Groupes & $(377)$ & $(94)$ & $(99.4)$ & $(164)$ & $(591.1)$ & 86.9 \\
n=15 & $(180.3)$ & $(33.6)$ & $(77)$ & $(67.1)$ & $(334.8)$ & 167.3 \\
\hline Total & 170.3 & 69.3 & 55.8 & 72.8 & 368.2 & \\
$\mathbf{n}=36$ & $(323.8)$ & $(79.2)$ & $(91)$ & $(135.4)$ & $(526.7)$ & \\
\hline
\end{tabular}

Source : élaboration propre 
Rapportée à la taille de chaque ménage (voir tableau 3), la dépense moyenne par personne est de $179.1 €$ pour la catégorie des individuels séjournant 3.3 jours sur la commune du Guilvinec. L'estimation de la catégorie des groupes atteint 86.9 euros pour un séjour généralement inférieur à la journée (0.7 jours). On peut avancer des coefficients budgétaires dans la dépense totale des visiteurs en quatre postes. L'hébergement représente $49 \%$ de l'ensemble des dépenses chez les individuels, contre 32\% pour les groupes. La catégorie autres loisirs (comprenant l'achat de souvenirs) est identique, $20 \%$ pour les individuels et $21 \%$ pour les visiteurs en groupes. C'est sur la restauration et l'achat de produits de la mer que les coefficients s'écartent. Les individuels, en séjour de plus longue durée, consacrent $18 \%$ de leur revenu en restauration et 13\% pour l'achat de produits de la mer. Les visiteurs en groupe, de passage sur une seule journée ou demi-journée, affectent un quart de leurs dépenses sur chacun de ces deux postes.

La visite d'Haliotika se décline comme une combinaison de biens (visite du centre, visite de la criée, atelier cuisine, découverte de l'estran, sortie en mer) sans toutefois la possibilité d'un consentement à payer plus cher. La préférence pour la consommation de produits locaux associés à une démarche de qualité peut être déclenchée par cette visite, notamment par les dépenses de restauration, d'achat de produits de la mer, de souvenirs, voire dans l'hébergement en gîtes (Mollard et al., 2006).

En 2016, 32184 personnes ont fréquenté Haliotika individuellement auxquels s'ajoutent 6247 passages au sein de groupes. Ces visiteurs ont donc d'après l'enquête apporté aux commerces, restaurants et hébergeurs de la commune du Guilvinec un revenu de 6,3 millions d'euros $(32184 x 179,4+6247 \times 86,9)$. Les visiteurs individuels apportent $91 \%$ de ce flux monétaire, en rappelant la limite forte que les dépenses des touristes et excursionnistes au mois de juin ne reflètent pas la population sur la période d'ouverture.

Une limite forte, énoncée à la section précédente, porte sur le statut du visiteur et son attachement au territoire d'étude. Sur la base de l'échantillon restreint aux visiteurs déclarant des dépenses sur la commune (36 individus), on soutient l'hypothèse d'une base territoriale communale. L'extrapolation à l'ensemble de la population semble donc surestimée car 36\% ((5736)/57) des répondants à la seconde enquête ne déclarent aucune dépense sur la commune. En considérant une base territoriale étendue au territoire de Cornouaille, sur lequel réside la majorité des visiteurs, l'estimation globale est alors plus proche de l'état réel des dépenses touristiques portant intérêt au centre de découverte des métiers de la pêche. Naturellement, les flux monétaires (hébergement, restauration, visites) sont plus diffus avec un effet qui ne peut être rapporté à la seule commune du Guilvinec.

Cette première estimation issue de l'enquête permet de comparer les dépenses locales d'une population touristique à la valeur des débarquements issus de la pêche sur le territoire. Le flux des dépenses touristiques, estimé dans cet article à 6,3 millions d'euros, représente près de $6 \%$ du chiffre d'affaires des produits de la mer débarqués sur l'ensemble des ports appartenant au quartier maritime (Le Guilvinec, Saint-Guénolé, Lesconil, Loctudy). Rapporté à la valeur de la baudroie et la langoustine, la somme des flux touristiques représente respectivement $22 \%$ et $30 \%$ du chiffre d'affaires de ces deux espèces cibles (IFREMER, 2017). Certes, une partie des dépenses de la clientèle touristique intégrant la visite d'Haliotika dans leur séjour ne profite pas intégralement au territoire. Dans une interprétation keynésienne, la demande de produits importés peut être forte. 
Une part des hébergements et la restauration hors produits locaux, peuvent échapper à l'économie locale. Une analyse menée sur les emplois liés au tourisme littoral et à la pêche est une autre occasion de rapprocher les deux secteurs, au moins comme premiers éléments de présentation.

Le recensement de la population de marins sur le quartier maritime du Guilvinec en 2016 indique 646 postes embarqués (IFREMER, 2017). La masse salariale globale sur ce territoire se situe entre 20 et 40 millions d'euros pour un chiffre d'affaires de 110 millions d'euros. Le tourisme littoral représente certainement un secteur important d'activité économique locale même s'il semble difficile d'avoir une idée exacte de son poids économique, du nombre d'emplois en équivalent temps plein (DOSDAT et MOULINIER, 2014). Les statistiques collectées par la Chambre de Commerce de Quimper-Cornouaille confirment que ce territoire s'impose comme la première zone touristique du Finistère, avec plus de $52 \%$ des capacités d'hébergements touristiques (CHAMBRE DE COMMERCE ET D'INDUSTRIE, 2014). Le nombre d'hôtels, cafés et restaurants y est également élevé (1662 établissements inscrits, dont plus de 858 restaurants) générant 3141 emplois directs en 2012.

Rappelant les limites de l'enquête réduite à la fréquentation du centre Haliotika sur le mois de juin, le flux des dépenses touristiques est loin d'être négligeable.

Si l'objectif est de fournir une première estimation des dépenses directement et indirectement liées à l'attraction touristique liée aux pêches maritimes, nous rappelons les limites d'un tel exercice. On identifie deux principales limites pouvant mettre en question les estimations fournies. La taille de l'échantillon et la périodicité est un premier facteur réduisant la portée générale de l'exercice. La couverture estivale, sur juillet et août, apporterait des changements structurels dans les caractéristiques sociodémographiques. Le second facteur limitant porte sur le statut du visiteur, posant trois questions soulevées à la précédente section (6.2. Enquête sur les dépenses au cours du séjour). Séparé les excursionnistes (sans nuitée déclarée hors de leur résidence principale) des touristes est une étape supplémentaire à intégrer au questionnaire, sous réserve d'étendre la période d'enquête. La seconde question, intégrée dans le questionnaire, consiste à distinguer les touristes séjournant sur la commune et hors de la commune. Un taux d'échantillonnage plus poussée que celui atteint dans cette recherche permettrait de consolider les résultats, principalement sur les dépenses du séjour des touristes en résidence sur la commune. La troisième question cerne l'attractivité du site touristique et les multi-motivations de la clientèle. Une étude de notoriété, à intervalle régulier, doit pouvoir préciser la position du site touristique dans les préférences des consommateurs.

L'estimation des dépenses touristiques des visiteurs d'Haliotika fournit un seuil de référence qui doit être replacé sous les limites de l'enquête. Conformément à la méthodologie d'évaluation des retombées économiques, celles-ci se déclinent exclusivement sur les déclarations des non résidents. On rappelle que l'échantillon est constitué exclusivement de non résidents, soit une demande extérieure à la commune. En revanche, le flux de dépenses n'intègre pas la valeur nonmarchande, telle que la valorisation du patrimoine maritime local, pour la population résidente. Une autre limite forte réside dans la période de la collecte des données, en pré-saison estivale. Juillet et août représente $40 \%$ du flux de visiteurs, pour $10 \%$ en juin. Le profil sociodémographique et le comportement des dépenses en forte activité estivale ne peuvent être associés à celui des visiteurs de juin et septembre. 
Les trajectoires de croissance de l'industrie de base (les pêches maritimes) et de l'industrie induite (le tourisme littoral) n'affichent pas les mêmes limites. Soumises à l'objectif d'un rendement soutenable, les pêches maritimes européennes ont l'obligation de limiter la pression de pêche face à l'épuisement des ressources. L'industrie du tourisme, sous réserve là-aussi de gérer la capacité d'accueil, offre un potentiel de développement moins contraint. L'articulation des deux industries autour d'un panier de biens, comme celui offert par le centre de découverte des pêches maritimes, crée des opportunités pour le développement territorial. On propose trois points de discussion sur ce thème.

\section{$-8-$}

\section{Discussion et conclusion}

Les éléments rassemblés autour du thème du caractère territorial des attractions touristiques liées aux pêches maritimes sont (i) la spécificité forte du Guilvinec par les emplois de la sphère productive, (ii) la tendance à la hausse de la fréquentation d'Haliotika, (iii) l'offre d'un panier de biens composites à Haliotika, (iv) une première estimation des dépenses touristiques des visiteurs du centre. Trois points de discussion sont proposés sur la base de ces éléments. A quel modèle du panier de biens correspond le cas d'étude retenu ? Quel est le territoire pertinent pour définir le rayonnement du centre de découverte des pêches maritimes ? Où se situe la rente de qualité territoriale?

A quel modèle du panier de biens correspond le cas d'étude retenu ? Les interdépendances entre pêche et tourisme sont bien réelles, et peuvent même inverser le lien causal entre une industrie de base, la pêche, et l'industrie induite, le tourisme, lorsque la demande pour des produits de qualité tirés de pêcheries côtières assure des revenus élevés aux pêcheurs. Le modèle du panier de biens adapté aux produits de la mer emprunte aux quatre variantes proposées dans la littérature. En effet, l'émergence récente d'un nouveau paradigme des pêches plaçant la protection de l'écosystème comme objectif prioritaire (PIKITCH et al., 2004) conduit les Etats à créer des aires marines protégées (modèle 1). Ce nouveau paradigme inscrit dans la dernière réforme de la Politique commune de la pêche (PARLEMENT EuropéEn et CONSEIL DE L'UNION EuROPÉENNe, 2013) encourage une plus forte implication des parties prenantes dans une coordination des actions publiques et privées (modèles 3 et 4), notamment par des modes de gestion décentralisés (modèle 3).

Quel est le territoire pertinent pour définir le rayonnement du centre de découverte des pêches maritimes ? Le choix du territoire pertinent dans l'étude de l'attractivité du centre Haliotika penche sur l'ensemble de la Cornouaille, plutôt que le quartier maritime ou la commune du Guilvinec. Le comité régional du tourisme en Bretagne définit dix grandes destinations avec un découpage géographique distinct des limites départementales. La Cornouaille est l'une d'entre elles, dotée d'une légitimité administrative depuis la loi de 1999 sur l'orientation pour l'aménagement et le développement durable du territoire. Les ports de pêche situés sur ce territoire de Cornouaille (Douarnenez, Audierne, Saint-Guénolé, Guilvinec, Lesconil, Loctudy, Concarneau) dépendent d'une gestion unique par la Chambre de Commerce. C'est donc à cette échelle que le processus de développement territorial, autour du centre de découverte Haliotika, doit prendre appui. 
Où se situe la rente de qualité territoriale -RQT- ? La RQT se manifeste dans les achats de produits de la mer, commercialisés dans une démarche de valorisation (FONNER and SYLVIA, 2015). Les organisations professionnelles à la pêche, soutenues par les collectivités, ont tentées à plusieurs reprises d'inscrire leurs productions dans un segment de marché. Les initiatives les plus convaincantes sont généralement circonscrites à un territoire spécifique (bar de ligne de la pointe d'Audierne, sardine de Douarnenez, langoustine des ports du territoire de Cornouaille, CoquilleSaint-Jacques en baie de Saint-Brieuc ou de la rade de Brest). Le centre de découverte Haliotika, décrit sous la forme d'un premier panier de biens (visite du centre, visite de la criée, atelier cuisine, découverte de l'estran, sortie en mer), ne produit pas directement une rente attachée à la qualité des biens et au territoire. Il offre une première expérience dont l'un des attendus est de soutenir la production du territoire avec un consentement à payer plus élevé. Cela se vérifie au cours du séjour touristique sans certitude d'un effet prolongé sur les lieux de résidence principale des vacanciers. C'est ici une question de débat sur le modèle du panier de biens revisité. En effet, la RQT n'est déclarée qu'en observant un acte d'achat prolongé du vacancier hors des limites de production du bien ou service, notamment sur son lieu de résidence principale. Une stratégie d'exportation de la production locale, hors des limites spatiales du territoire d'origine de l'offre composite, est-elle possible sans détruire les valeurs cognitives (culture, patrimoine, identité) ? La stratégie d'exportation est-elle compatible avec les attributs du panier de biens souvent liée à une commercialisation en circuit court ou un commerce de proximité ? La rente liée à la qualité exprime une différenciation du produit que le consommateur peut identifier par un label ou un mode de commercialisation. En revanche, la rente procurée par un attachement au territoire exige de bien définir les limites spatiales. L'évaluation d'une RQT repose sur des entretiens auprès des consommateurs susceptibles d'accorder une valeur monétaire plus élevée à des produits de la mer dont le mode de commercialisation identifie clairement le lieu de production et le patrimoine historique et culturel comme valeurs intrinsèques du produit. L'estimation du consentement à payer des consommateurs n'était pas l'objet de l'article, mais c'est une problématique qui lui fait écho. En principe, une RQT doit être dégagée dès lors qu'une stratégie territoriale de long terme aboutit à produire une offre de biens ou services composites situés en un même lieu (PECQUEUR, 2001). Le rôle du centre de découverte s'exerce en amont en faisant découvrir le panier de biens territorialisé. Une évaluation du consentement à payer des produits de la mer débarqués dans les ports de pêche de Cornouaille, base territoriale retenue, est une suite logique de cette recherche. 


\section{Remerciements}

Les auteurs adressent leurs vifs remerciements aux relecteurs pour leurs commentaires et suggestions. Cette recherche a bénéficié du soutien financier de la Fondation de France.

\section{Références bibliographiques}

ACHESON JM (1975) The Lobster Fiefs: Economic and Ecological Effects of Territoriality in the Maine Lobster Industry. Human Ecology 3(3) : 183-207.

ALBAN F (1998) Pêche professionnelle et activités récréatives. Examen des potentialités de développement d'une pluri-activité : Le cas de la mer d'Iroise. Mémoire de DEA, Université de Bretagne Occidentale, Brest.

ALBAn F, BONCOEUR J (2004) An Assessment of the Potential Interest of Fishermen to Engage in Boat-Chartering in the Context of a Marine Park: the case of the Iroise Sea, 185-203. In : BOISSEVAIN J. et SELWYN T. (eds) Contesting the foreshore: tourism, society, economics and politics of the coast. MARE Publication Series, Centre for Maritime Research, Amsterdam University Press, Amsterdam.

ALBAN F, BONCOEUR J, RONCIN N (2011) Assessing the impact of mpas on society's well-being: an economic perspective. in : CLAUDET J. (ed.) Marine protected areas: effects, networks and monitoring - a multidisciplinary approach, Cambridge University Press.

ARNAL C (2012) L'agriculture, élément de la qualité de vie des villes moyennes. Revue d'Economie Régionale et Urbaine, 2 : 245-264.

BAZIN-BENOÎT S, BECKERICH C, DELAPLACE M (2014) Valorisation touristique du patrimoine et dessertes TGV. Le cas de quatre villes intermédiaires proches de Paris. Revue d'Economie Régionale et Urbaine, 5 : 865-883.

BOJAMIC D-C, CALANTONE R-J (1990) A contribution approach to price bundling in tourism. Annals of Tourism Research, 17 : 528-540.

BOULARD J-C (1991) L'épopée de la sardine, un siècle d'histoires de pêches. Editions OuestFrance, Ifremer, Rennes.

CALLON M (1986) Some elements of a sociology of translation: domestication of the scallops and the fishermen of St-Brieuc bay (196-223). in : LAW J., Power, action and beliefs: a new sociology of knowledge?, Londres, Routledge.

CANADA J-S, MUCHNIK J (2011) Introduction : ancrage et identité territoriale des systèmes agroalimentaires localisés. Economie Rurale, 322 : 4-10.

CARLSEN J, WOOD D (2004) Assessment of the economic value of recreation and tourism in western Australia's national parks, marine parks and forests, technical report, Townsville, Sustainable tourism cooperative research center, 29p. 
CHAMBRE DE COMMERCE ET D'INDUSTRIE (2014) Atlas économique de Cornouaille, Quimper, $112 p$.

CHARLES E (2009) eco-labelling : A new deal for a more durable fishery management ? Ocean Coastal Management 52 : 250-257.

CHARLES E, THOUEMENT H (2007) Le label territorial, facteur d'attractivité touristique: une étude appliquée à la Bretagne. Téoros Revue de recherche en tourisme 26(2) : 33-38.

CONFOLENT D (2007) De la marine aux affaires maritimes. Les archives orales du ministère, http://www.archives-orales.developpement-durable.gouv.fr.

CORDON F (1950) Les invalides de la marine. Une institution sociale de louis XIV. Son histoire, de Colbert à nos jours. Edition SEGMC, paris, 254p.

DANIEL H, ALLEN T, BRAGG L, TEISL M, BAYER R, BILLINGS C (2008) Valuing lobster for Maine coastal tourism: Methodological considerations. Journal of Food Service, 19 : 133-138.

DAUMAS J-C (2007) Districts industriels : du concept à l'histoire - les termes du débat. Revue Economique, 1(58) : 131-152.

DELAMARE A (2010) Diversification socio-économique des secteurs de la pêche et des cultures marines sur la façade Aglia. Rapport Aglia, http://www.aglia.fr/images/projet/article/14/pdf/106rapport_diversification_vf.pdf

DELBOS G, PREMEL G (1996) The breton fishing crisis in the 1990s: Local society in the throes of enforced change in fisheries management in crisis. in : CREAM K. and SYMES D., Fisheries management in crisis, Oxford, Fishing news books.

DOSDAT A, MOULINIER H, (2014) Economie maritime en bretagne : changeons de regard, Conseil Economique, Social et Environnemental de Bretagne, Rennes, 199p.

DURAND M-H (1991) La crise sardinière française : les premières recherches scientifiques autour d'une crise économique et sociale. in : CURY P., ROY C. (eds), Pêcheries Ouest Africaines: variabilité, instabilité et changement. Orstom, Paris.

FELDMAN M-P, AUDRETSCH D-B (1999) Innovation in cities: science-based diversity, specialization and localized competition. European Economic Review, 43 : 409-429.

FONNER R, SYLVIA G, (2015) Willingness to pay for multiple seafood labels in a niche market. Marine Resource Economics, 30(1) : 51-70.

FOUCAUD F (2008) Le pescatourisme, un complément d'activité pour les marins pêcheurs. Espaces $261: 3$.

FRANCEAGRIMER 2014 Données de vente déclarées en halles à marée en 2013, Paris, 96p.

GAGNON S (2007) L'attractivité touristique des territoires. Téoros, Revue de recherche en tourisme 26(2) : 3-11.

GOUZIEN A (2014) Approche socio-anthropologique de la complexité territoriale: les territoires de la pêche maritime en Bretagne sud. Communication au séminaire du 16 janvier 2014. axe1 développement et société, CIAPHS. 
GUILLOTREAU P (1992) Le méso-système halio-alimentaire européen, analyse et mode de fonctionnement, thèse de doctorat, Université de Rennes 1.

HIRCZAK M, MOALLA M, MOLLARD A, PECQUEUR B, RAMBONILAZA M, VOLLET D (2008) Le modèle du panier de biens. Economie Rurale, 308 : 55-70.

HOLBROOK M-B, HIRSCHMAN E-C (1982) The experiential aspects of consumption: consumer fantasies, feelings and fun. Journal of Consumer Research, 9 : 132-140.

IFREMER (2017) Activité des navires de pêche 2016, quartier maritime Guilvinec, http://sih.ifremer.fr/content/download/30460/205628/file/guilvinec\%202016.pdf

INSEE (2014) Les dépenses des touristes en France. Insee première, n¹510, juillet 2014.

INSEE (2015) Sphère présentielle et sphère productive, guide méthodologique, https://www.insee.fr/fr/statistiques/fichier/.../spheres_guide_methodologique.pdf

KOSIANSKI J-M, MONINO J-L (2005) Les grandes expositions du musée de Lodève, facteur de développement local. in: Musées \& Tourisme, Editions touristiques européennes. Les cahiers Espaces, Paris 87.

KREZIAK D, LACROIX A, LENGLET F (2012) Les produits de terroir : vecteurs de valorisation des aménités ? Revue d'Economie Régionale et Urbaine, 5 : 831-853.

LANCASTER KJ (1966) A new approach to consumer theory. The Journal of Political Economy 74 (2) : 132-157.

LE FLOC'H P, WILSON J (2017) Les pêches maritimes françaises, 1983-2013. Presses universitaires de Rennes, 213p.

LE FLOCH P, WILSON J, NASSIRI A (2017) Diversification after resource crises; the case of tourism in the french archipelago of Saint-Pierre and Miquelon. Canadian Journal of Regional Science 40(3) : 225-236.

LEIPER N, (1990) Tourist attraction systems. Annals of Tourism Research, 17 : 367-384.

LEON A, SAUVIN T (2010) L'entreprise et son empreinte territoriale : quelle politique d'attractivité. Mondes en Développement, 149(1) : 43-52.

LEONARDI S, BEGOT E, LE GRAND C et al (2010) Etude sur l'état des lieux de la pêche professionnelle dans le parc naturel marin d'Iroise, Plouzané, 119p.

LESUEUR M, BOUDE J-P, LE GOFFE P (2007) La multifonctionnalité des pêches maritimes et des cultures marines à la lumière de la multifonctionnalité de l'agriculture. Agrocampus Rennes, 33 p., http://halieutique.agrocampus-ouest.fr/pdf/813.pdf

LESUEUR M, PICAULT D, BENOIT J et al (2014) Analyse des retombées économiques induites par le secteur de la pêche dans l'économie locale - le cas d'étude de la fête de la coquille Saint-Jacques à Erquy. Les publications du pôle halieutique Agrocampus ouest n²7, 36p.

MAILLEFERT M, ROBERT I (2017) Nouveaux modèles économiques et création de valeur territoriale autour de l'économie circulaire, de l'économie de la fonctionnalité et de l'écologie industrielle. Revue d'Economie Régionale et Urbaine, 5 : 905-934. 
MARTÍNEZ-ESPIÑEIRA R, AMOAKO-TUFFOUR J (2009) Multi-destination and multi-purpose trip effects in the analysis of the demand for trips to a remote recreational site. Environmental Management, 43 : 1146-1161.

MERRIEN V, LESUEUR M, BOUDE J-P, FOLLIARD G (2008) Diversification des activités de pêche en Bretagne. Agrocampus ouest, http://halieutique.agrocampus-ouest.fr/pdf/641.pdf

MOLLARD A (2001) Qualité et développement territorial : une grille d'analyse théorique à partir de la rente. Economie Rurale, 263 : 16-34.

MOLLARD A, RAMBONILAZA M, VOLLET D (2006) Aménités environnementales et rente territoriale sur un marché de services différenciés: le cas du marché des gîtes ruraux labellisés en France. Revue d'Economie Régionale et Urbaine, 2 : 251-276.

MORANDIERE DE LA C (1967) La pêche française de la morue à Terre-Neuve, du XVIème siècle à nos jours : son importance économique, sociale et politique. Contributions du centre d'études arctiques et finno-scandinaves, ${ }^{\circ}$ 4, Ecole Pratique des Hautes Etudes, Sorbonne.

PARLEMENT EUROPÉEN ET CONSEIL DE L'UNION EUROPÉENNE, (2013) Règlement (ue) $n^{\circ} 1380 / 2013$ du Parlement européen et du Conseil du 11 décembre 2013 relatif à la politique commune de la pêche, modifiant les règlements (ce) $n^{\circ} 1954 / 2003$ et (ce) $n^{\circ} 1224 / 2009$ du Conseil et abrogeant les règlements (ce) $n^{\circ} 2371 / 2002$ et (ce) $n^{\circ}$ 639/2004 du Conseil et la décision 2004/585/ce du conseil, Bruxelles.

PARSONS S-L (1995) La gestion des pêches maritimes au Canada. Conseil national de recherches, Ottawa.

PECQUEUR B (2001) Qualité et développement territorial : l'hypothèse du panier de biens et de services territorialisés. Economie Rurale 161 : 37-49.

PERON F, MARIE G (2010) Atlas du patrimoine maritime du Finistère. Editions Le Télégramme, Brest.

PIKITCH E-K, SANTORA C, BABCOCK E-A, et al (2004) Ecosystem-based fishery management. Science, $305: 346-347$.

POCHEAU C, (2017) Analyse des interdépendances entre la pêche et le tourisme : haliotika la cité de la pêche au guilvinec. mémoire de master 2, ingénieur sciences halieutiques et aquacoles, Agrocampus ouest, Rennes, 60p.

REQUIER-DESJARDINS D (2010) L'évolution du débat sur les SYAL : le regard d'un économiste. Revue d'Economie Régionale et Urbaine, 4 : 651-668.

RONCIN N, ALBAN F, CHARBONNEL E et al (2008) Uses of ecosystem services provided by MPAs : how much do they impact the local economy? A southern Europe perspective. Nature Conservation $16: 256-270$.

ROPARS-COLLET C, LEPLAT M, LE GOFFE P, LESUEUR M (2015) La pêche professionnelle est-elle un facteur d'attractivité récréative sur le littoral ? Revue Economique 66(4) : 729-754.

SCHRANK WE (2005) The New-Foundland fishery: ten years after the moratorium. Marine Policy $29: 407-420$. 
VAUCLARE C (1987) Naissance d'une industrie. in: MOllat M., (ed.), Histoire des pêches maritimes en France, Editions Privat, Toulouse.

VOLTAIRE L, LEVI L, ALBAN F, BONCOEUR J (2017) Valuing cultural world heritage sites: an application of the travel cost method to Mont-Saint-Michel. Applied Economics, 49(16): 15931605.

ZIMMERMANN B (2005) Entreprises et territoires: entre nomadisme et ancrage territorial. La Revue de l'Ires, 47(1) : 21-36. 